\title{
Analysis on Fluid Characteristics of Friction Plate in Wet Multi-plate Clutch Based on Computational Fluid Dynamics
}

\author{
Liying Miao1, Xiusheng Cheng1, Zhonghua Liu2, Xuesong Li1 \& Chuanfang \\ Zhang3
}

1 State Key Laboratory of Automotive Simulation and Control, Jilin University, Changchun, China 2 China FAW Co., Ltd R\&D Center, Changchun, China

3 Beijing Hainachuan Automotive Parts Co., Ltd, Beijing,, China

Keywords: flow characteristic, convective heat transfer, wet multi-plate clutch, simulation analysis.

\begin{abstract}
In order to study the fluid characteristics of wet clutch in the process of engagement, the flow characteristics of radial oil grooves between the friction plates were simulated by Fluent, and the different entrance flow rates and different entrance temperature of heat exchanger performance were also discussed. The simulation results were compared with the test results, showing that the accuracy of the simulation calculation method. The calculation results can be used to study and optimal design the friction characteristics of the wet clutch.
\end{abstract}

\section{Introduction}

The wet clutch may produce a lot of heat in the process of engagement. If the temperature of friction plates is too high, the friction plates will be warped and cause permanent failure, thus reducing the service life of the clutch[1]. At the same time, the temperature change during the engagement process of the wet clutch affect the coefficient of friction, caused jitter and seriously affecting the vehicle comfort. Therefore, the research on the flow and heat transfer performance of cooling oil between the friction plates was crucial to maintain a good friction characteristics. This paper based on CFD platform, made the numerical simulation on the flow field characteristics of wet clutch, and obtained the flow characteristics and the convective heat transfer performance of different entrance flow and different entrance temperature.

\section{The establishment of the model}

The wet clutch is mainly composed of drive and driven friction plates. When clutch engaged, under the control of the hydraulic, the friction torque is transmitted by the friction of the friction plates. Fig.1 is the wet clutch for research.

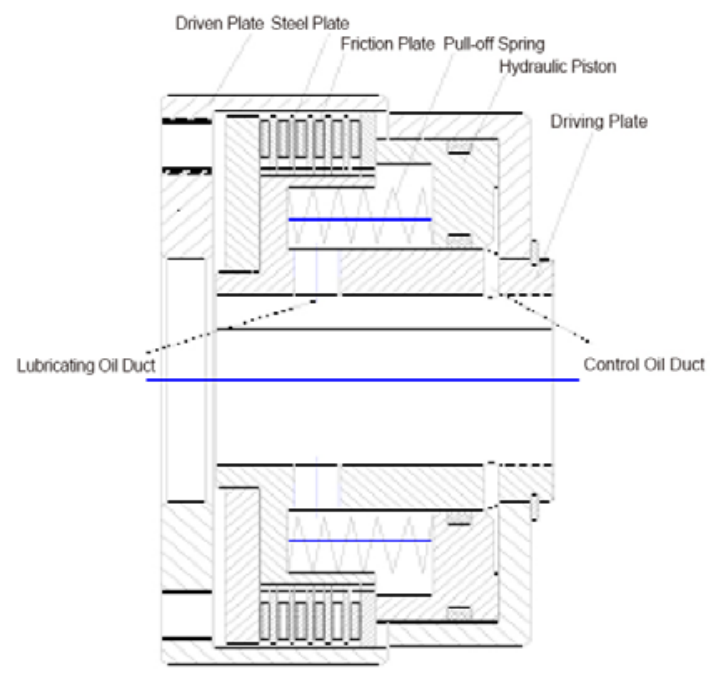

Figure 1: Profile of Wet Multi-disc Clutch. 
A pair of friction piece and dual steel piece were called a pair of friction pair, because of the symmetry of the structure of the wet clutch friction pair and the load, it is enough to pick up just a pair of friction pair to analyze[2,3,4]. Ignore the influence of the dual steel chip and friction plate tooth, friction pair structure diagram was shown in fig.2.

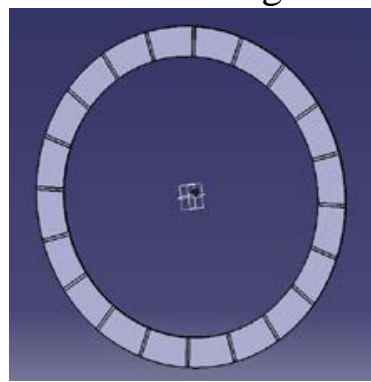

(a) friction piece

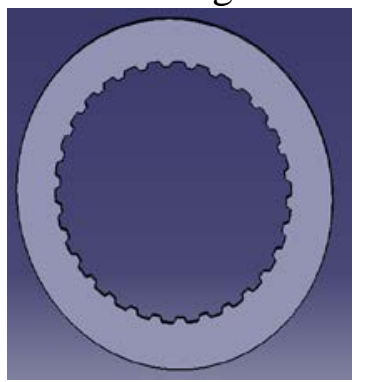

(b) dual steel piece

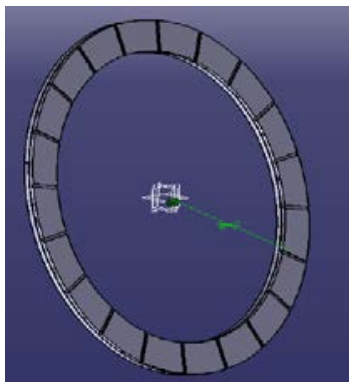

(c) assemble

Figure 2: Friction pair structure.

According to the theory of heat transfer, the heat conduction equation of the friction pair under rectangular coordinate system is:

$$
\frac{\rho_{\mathrm{i}} \cdot \mathrm{c}_{\mathrm{i}}}{\lambda_{\mathrm{i}}} \frac{\partial T_{\mathrm{i}}}{\partial \mathrm{t}}=\frac{\partial}{\partial \mathrm{x}}\left(\frac{\partial T_{\mathrm{i}}}{\partial \mathrm{x}}\right)+\frac{\partial}{\partial \mathrm{y}}\left(\frac{\partial T_{\mathrm{i}}}{\partial \mathrm{y}}\right)+\frac{\partial}{\partial \mathrm{z}}\left(\frac{\partial T_{\mathrm{i}}}{\partial \mathrm{z}}\right)
$$

Among the equation, $\lambda_{\mathrm{i}}$ is the coefficient of thermal conductivity, $\rho_{\mathrm{i}}$ is density, $\mathrm{c}_{\mathrm{i}}$ is specific heat, $T_{\mathrm{i}}$ is the temperature of the friction element, and $\mathrm{T}$ is slipping time, $\mathrm{x}, \mathrm{y}, \mathrm{z}$ mean friction pair some point coordinates, $\mathrm{i}=1,2$. In this paper, subscripts 1,2 correspond to dual steel piece and friction respectively, Don't consider the effect of material wear and think that sliding friction work into frictional heat. Heat flow density on one point of contact surface of friction pair satisfies the follow equation.

$$
\mathrm{q}(\mathrm{x}, \mathrm{y}, \mathrm{z})=\mu(\square \mathrm{w}) \cdot \mathrm{p}_{\mathrm{c}}(\mathrm{x}, \mathrm{y}, \mathrm{z}, \mathrm{t}) \cdot \square \omega(\mathrm{t}) \cdot \mathrm{r}
$$

Among the equation, $\mathrm{q}$ means the heat flux at some point on per unit area of friction pair, $\mathrm{p}_{\mathrm{c}}$ means the contact pressure of contact point for friction pair, $\square \mathrm{w}$ means the elative angular velocity difference between friction piece and dual steel piece, $r$ is the distance from the center of friction pair. Contact surface engenders relative sliding friction heat, heat flux between the lining and the dual contact surface of the steel are allocated, set the input eqn (1) and eqn (2) to the friction piece and dual steel piece of heat flux density respectively, as showed in eqn (3) and eqn (4):

$$
\begin{gathered}
\mathrm{q}_{1}=\frac{K_{\mathrm{q}}}{K_{\mathrm{q}}+1} \mathrm{q}(\mathrm{x}, \mathrm{y}, \mathrm{z}, \mathrm{t}) \\
\mathrm{q}_{2}=\frac{1}{K_{\mathrm{q}}+1} \mathrm{q}(\mathrm{x}, \mathrm{y}, \mathrm{z}, \mathrm{t})
\end{gathered}
$$

Under the premise of temperature continuous on both side of the contact surface, the ratio between them can be express as eqn (5):

$$
\mathrm{K}_{\mathrm{q}}=\frac{\mathrm{q}_{1}(\mathrm{x}, \mathrm{y}, \mathrm{z}, \mathrm{t})}{\mathrm{q}_{2}(\mathrm{x}, \mathrm{y}, \mathrm{z}, \mathrm{t})}=\left(\frac{\lambda_{1} \cdot \rho_{1} \cdot \mathrm{c}_{1}}{\lambda_{2} \cdot \rho_{2} \cdot \mathrm{c}_{2}}\right)^{0.5}
$$

\section{Stimulation and results analysis}




\subsection{The inner flow field analysis}

The stimulation of fluid streamline figures of radial slots friction plate was shown in fig.3 and fig.4.

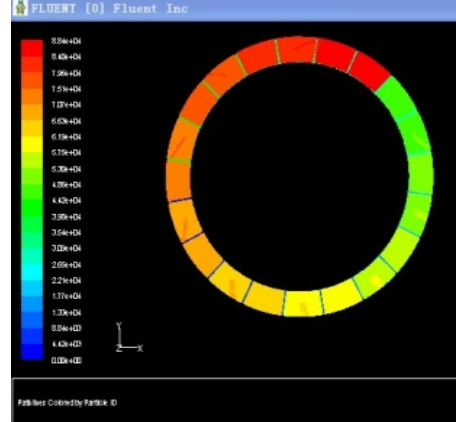

Figure 3: Fluid Streamline of radial slots friction plate.

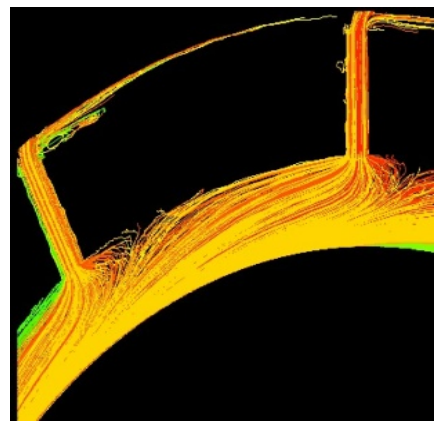

Figure 4: Magnification of fluid streamline.

A strong vortex emanate from the entrance of oil, because rotational speed was very high when friction pairs are preparing engaged, so oil acted upon by larger centrifugal force and shocks plate groove-walls then encounter obstacles, vortex was formed finally[5,6,9,10]. The oil acted upon by centrifugal force and entrance oil pressure simultaneously, the oil flows to friction plates outer edges through friction plate surface grooves.

The velocity vector figures of radial slots friction plate as shown in fig. 5 and fig.6. The oil acted upon by centrifugal force and entrance oil pressure, so it's two flow directions, one moving in centrifugal spin and another moving in radial flowing from the entrance to the outlet. The flow rate is increasing when oil flowing from the from the entrance to the outlet, and it's top flow rate of up to $12.7 \mathrm{~m} / \mathrm{s}$. As we can see in the figures, the flow rates in grooves are higher than others, such as the entrance, it is because that the exist here of vortex make the oil blocked, result in reducing the flow rate.

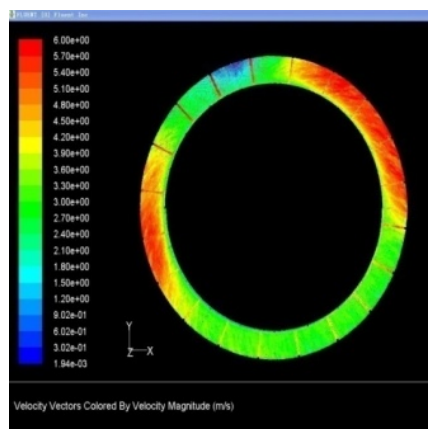

Figure 5: The velocity vector of radial slots friction plate. 


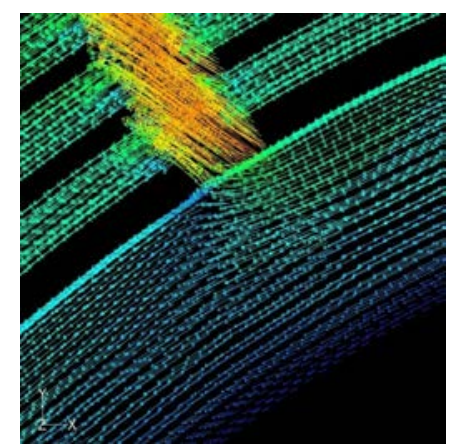

Figure 6: Magnification of the velocity vector.

\subsection{The heat convection simulation analysis}

By simulation analysis of heat convection conditions, using k-e viscosity model and Pressure-based solver of FLUENT software. Energy equations are added to the basic numerical calculating equations $[7,8,11]$. Set the flow rate of the entrance to $0.8 \mathrm{~m} / \mathrm{s}$, the clutch speed to $1000 \mathrm{rpm}$, and the oil temperature of the entrance to $300 \mathrm{k}$.

(1) Different inlet flow radial grooves lining convective heat transfer analysis

Fig.7 was the radial grooves friction plate convective heat transfer simulation when a fluid inlet velocity was in turn $0.5 \mathrm{~m} / \mathrm{s}, 0.7 \mathrm{~m} / \mathrm{s}, 0.9 \mathrm{~m} / \mathrm{s}, 1.0 \mathrm{~m} / \mathrm{s}$. Different inlet velocity corresponded to different clutch inlet flow.

As can be seen from fig.7, the friction surface the temperature is gradually lowered. The reason is that the greater the inlet velocity, the greater the corresponding clutch inlet flow, the more cooling fluid heat more fully, but also more conducive to heat the greater the flow rate.

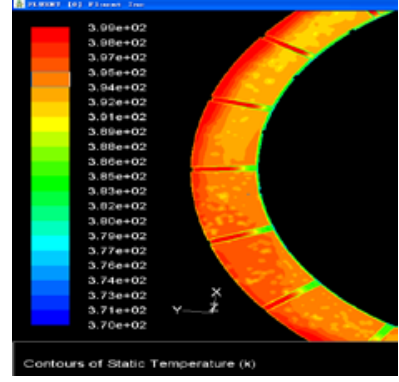

(a) $0.5 \mathrm{~m} / \mathrm{s}$ convective heat map

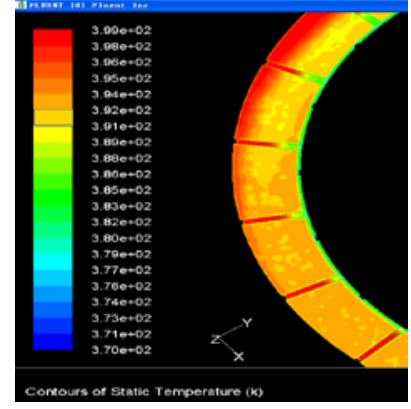

(c) $0.7 \mathrm{~m} / \mathrm{s}$ convective heat map

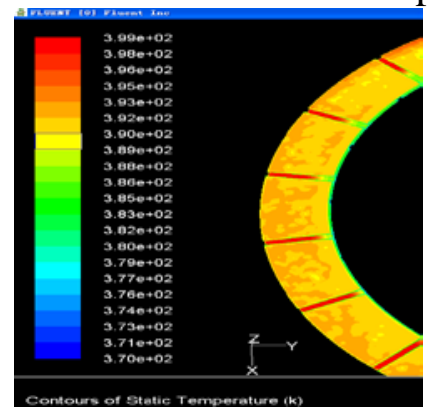

(e) $0.9 \mathrm{~m} / \mathrm{s}$ convective heat map

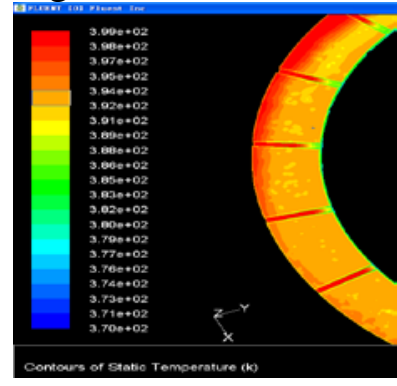

(b) $0.6 \mathrm{~m} / \mathrm{s}$ convective heat map

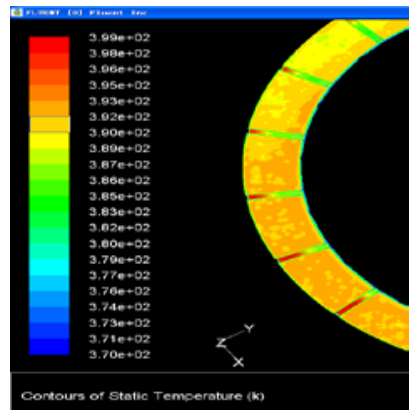

(d) $0.8 \mathrm{~m} / \mathrm{s}$ convective heat map

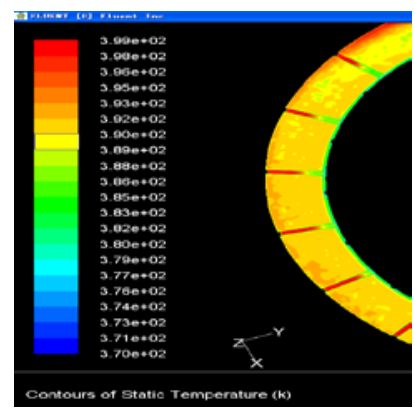

(f) $1.0 \mathrm{~m} / \mathrm{s}$ convective heat map

Figure 7: Different inlet velocity radial grooves lining convective heat map. 

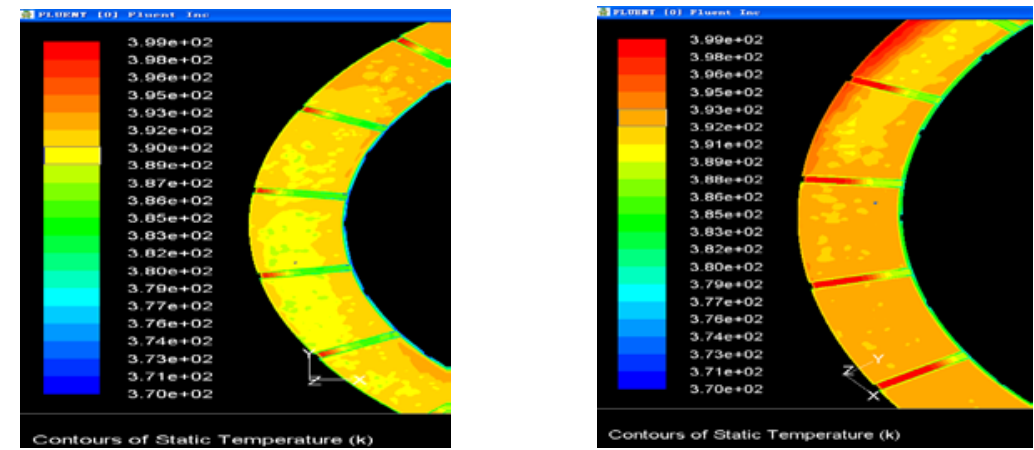

(a)Convective heat transfer figure of $300 \mathrm{~K}$

(b) figure of $310 \mathrm{~K}$
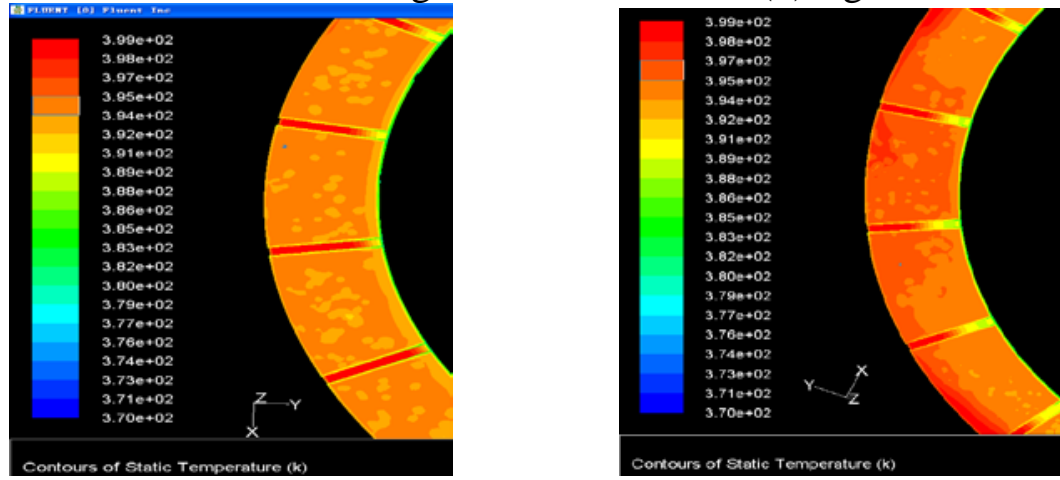

(c)Convective heat transfer figure of $320 \mathrm{~K}$

(d) figure of $330 \mathrm{~K}$

Figure 8: Convective heat transfer figure of different inlet temperatures.

(2) Convective heat transfer analysis of different fluid temperature at the inlet of the friction plate radial slots

As can be seen from fig.8, oil inlet temperature was in turn 300K, 310K, 320K, 330K, friction surface temperature gradually increased. The reason is the greater the inlet temperature is, the less sufficient the convective heat transfer is, the slower the friction surface temperature drops. If the temperature is too high, will cause the change of physical parameters of the oil that affect the heat transfer.

\section{Contrast of the experiment and simulation results}

For radial slot friction plate with a fixed input speed, change the inlet flow conditions for numerical calculation, and get the drag torque of the clutch, as shown in fig.9.Compare the simulation results with the experiment, as shown in fig.10.The comparison of the two curves shows that the simulation data is slightly smaller than the experimental data, because of the separation of the clutch in the bench test is not very thorough, and the friction gap is uneven. As can be seen from the trend of the two curves, with the increase of the inlet flow, drag torque has also been increased, they are positively related. Although the simulation curve has some difference with the experiment curve, their trend are the same, verified the accuracy and reliability of numerical calculation. 


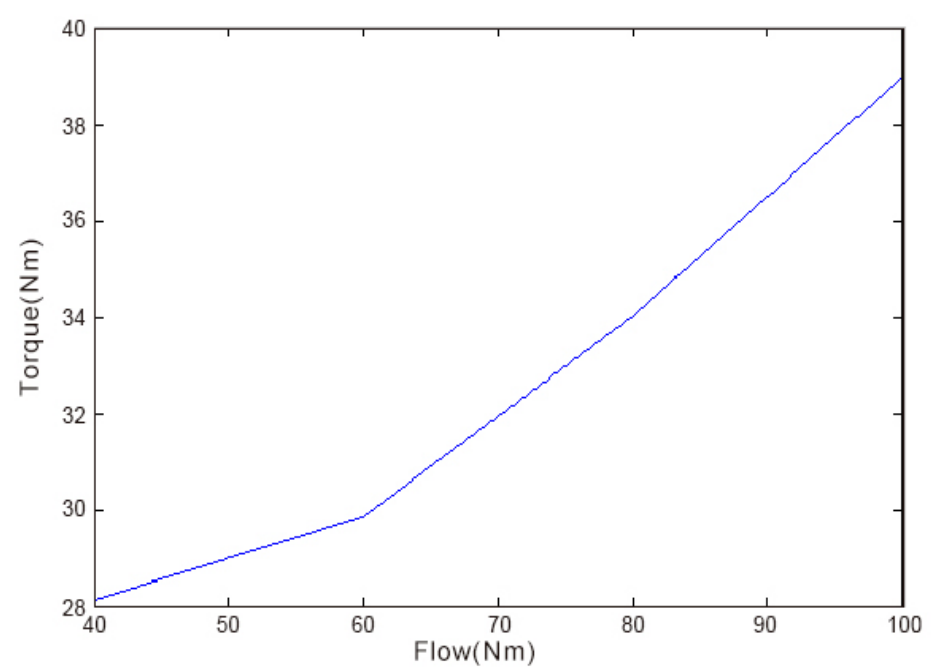

Figure 9: Drag torque experiment curve.

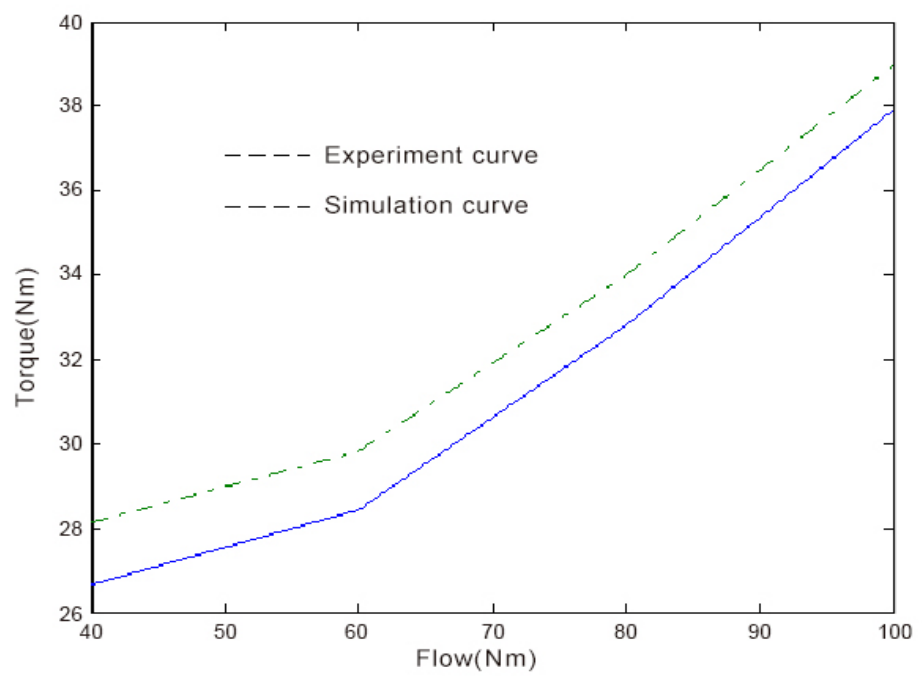

Figure 10: Simulation compare with experiment.

\section{Conclusion}

A three-dimensional model of a wet multi-plate friction clutch radial grooves was established, the flow field and the temperature field characteristics between the dual steel plate and the friction plate were simulated. The paper simulated convective heat transfer in the case of radial grooves friction plate for different inlet flow rate and inlet temperature. The numerical results were in good agreement with the experimental results, therefore, the software can be used to replace part of the test to calculate the wet clutch inner work flow analysis, quickly and economically optimize or design the wet clutch.

\section{Acknowledgment}

This work is supported by the National Nature Science Foundation of China (51305156).

\section{References}

[1].Zhang jinle, Ma Biao. Simulation on thermal characteristics of wet shifting clutch, Journal of Jilin University, 41(2):pp.321-326, 2011.

[2].Chen Yaofei. Study on the characteristics of wet multi plate clutch and the friction heat failure. Chongqing University, pp. 86-92, 2009.

[3].Sun Dongye, Hu Fengbing. Simulation and test on warpage characteristics of wet multi plate 
clutch, Journal of Chongqing University, 33(5):pp.1-6, 2010.

[4].Han Yong. Simulate and analysis on wet friction plates based on CFD technology. Jilin:Jilin University, pp. 35-39, 2011.

[5]. Zhu Hongqing. Study on heat and friction characteristics of wet clutch, Zhejiang University, pp. 67-72, 2012.

[6]. Zhang Shijun. Analysis on transient temperature field and oil road field of wet friction clutch. Chongqing University, pp. 43-54, 2009.

[7]Zhang Zhi-gang, Study on Several Working Characteristics of Wet Clutch, Zhejiang University, pp. 68-70, 2010.

[8] Feng Wei, Study on Starting and Shifting Control of Wet Double Clutch Transmission, Jilin University, pp. 40-61, 2010.

[9] Yinshu Wang, Xiusheng Cheng, Wei Feng, The Wet Clutch Pressure Control of Dual Clutch Transmission Based on FCMAC, edited by Applied Mechanics and Materials, v157-158, pp132-139, 2011.

[10] Cheng Yu, Research on Control Technology for Torque Assist Mechanism AMT Base on the Wet Clutch, Jilin University, pp. 105-112, 2014..

[11] J.Kim and S.Choi, Self-energizing Clutch Actuator System, FISTIA 2010 Congress, Budapest, 2010. 